\title{
Single Bilateral Dexamethasone Implant in Addition to Panretinal Photocoagulation and Oral Azathioprine Treatment in IRVAN Syndrome
}

\author{
Ali Osman Saatci Ziya Ayhan Ömer Takeş Aylin Yaman \\ F. Meltem Söylev Bajin \\ Department of Ophthalmology, Dokuz Eylül University, İzmir, Turkey
}

Key Words

Azathioprine $\cdot$ Dexamethasone implant $\cdot$ IRVAN syndrome $\cdot$ Laser photocoagulation · Ozurdex

\begin{abstract}
The idiopathic retinal vasculitis, aneurysms and neuroretinitis (IRVAN) syndrome is a disease characterized by multiple retinal macroaneurysms, neuroretinitis and peripheral capillary nonperfusion. Visual loss may result from either ischemia-related complications or macular involvement. Treatment is not always rewarding. We report a case with stage 2 IRVAN syndrome who was successfully treated with a single bilateral intravitreal dexamethasone implant in addition to panretinal photocoagulation and systemic azathioprine treatment.
\end{abstract}

(c) 2015 S. Karger AG, Basel

\section{Introduction}

The idiopathic retinal vasculitis, aneurysms and neuroretinitis (IRVAN) syndrome typically occurs in young patients and may produce multiple retinal macroaneurysms, neuroretinitis and peripheral capillary nonperfusion [1,2]. Samuel et al. [2] classified the disease into 5 stages: stage 1, macroaneurysms, exudation, neuroretinitis and retinal vasculitis; stage 2 , capillary nonperfusion as shown by fluorescein angiography; stage 3, posterior segment neovascularization of the disc or elsewhere and/or vitreous hemorrhage; stage 4, anterior 
Saatci et al.: Single Bilateral Dexamethasone Implant in Addition to Panretinal Photocoagulation and Oral Azathioprine Treatment in IRVAN Syndrome

segment neovascularization, and stage 5, neovascular glaucoma. Visual loss usually results from ischemia-related complications characterized with proliferative changes and/or from macular involvement including macular exudates and edema [3].

We report a case with stage 2 IRVAN syndrome who was successfully treated with a single bilateral intravitreal dexamethasone implant in addition to panretinal photocoagulation (PRP) and systemic azathioprine treatment.

\section{Case Report}

A 21-year-old woman was referred to us with bilateral blurred vision of 9 months' duration for further evaluation. She had received oral acetazolamide treatment beforehand with a presumed diagnosis of pseudotumor cerebri with a normal cranial MRI without any anatomic or functional benefit. Otherwise, her past medical and her family history were noncontributory. In December 2010, her best-corrected visual acuity (BCVA) was 20/25 in the right eye (OD) and 20/30 in the left eye (OS). She had full ocular motility, and her color vision, tested with Ishihara pseudoisochromatic plates, was good. Silt-lamp examination revealed occasional cells in the vitreous cavity in both eyes (OU). Both optic discs were hyperemic with dilated epipapillary arterioles in association with peripapillary exudates especially prominent in the OS (fig. 1a, b). Fluorescein angiography exhibited marked bilateral disc staining (fig. 1c, d). While the right optic coherence tomography (OCT) analysis was nearnormal (fig. 1e), there was subfoveal serous detachment with multiple hyperreflective dots corresponding to hard exudates in the OS (fig. 1f). Systemic evaluation and laboratory workup did not suggest any abnormality. No certain diagnosis could be established at that time, and the OS was treated with 1 bevacizumab and 3 consecutive intravitreal ranibizumab injections in a time span of 7 months in order to facilitate the resolution of serous retinal detachment. However, peripapillary serous retinal detachment remained unchanged.

The patient returned to our clinic in April 2013, almost 3 years after the last intravitreal injection. At this visit, the visual acuity was unchanged OU, but there were $360^{\circ}$ peripheral vasculitic changes and marked peripapillary exudates OU (fig. 2a, b). The fluorescein angiogram depicted severe $360^{\circ}$ peripheral capillary nonperfusion bilaterally (fig. 2c, d). On OCT examination, while the right macula looked still near-normal (fig. 2e), there was serous retinal detachment at the papillomacular area and multiple hyperreflective dots corresponding to hard exudates in the OS (fig. 2f). With these findings, the diagnosis of IRVAN was made. Treatment with azathioprine $(150 \mathrm{mg} /$ day $)$ was commenced. PRP was performed in three sessions approximately 2 weeks apart bilaterally. As the serous retinal detachment and peripapillary exudates did not change despite PRP, an intravitreal dexamethasone implant was administered in the OS in August 2013. The serous retinal detachment and peripapillary exudates decreased slowly after the implant in the OS. During the course, as the peripapillary exudates dramatically increased, an intravitreal dexamethasone implant was also administered in the OD in June 2014. No implant-related complication was noted in OU. At the last visit in November 2014, best-corrected visual acuity was 20/20 in the OD and 20/25 in the OS. Marked resolution of the peripapillary exudates was shown OU (fig. 3). 
Saatci et al.: Single Bilateral Dexamethasone Implant in Addition to Panretinal Photocoagulation and Oral Azathioprine Treatment in IRVAN Syndrome

\section{Discussion}

Treatment of the IRVAN syndrome is not always rewarding, and, thus, many therapeutic approaches have been utilized with partial success. These options include medical treatment, laser photocoagulation and vitreoretinal surgery, or a combination of them $[2,4-16]$.

PRP is the only well-accepted treatment modality in the presence of peripheral ischemia or neovascularization, and it should be employed as soon as possible to prevent ischemiadriven complications. PRP is often effective, especially in disease stages 2 and 3 [5-7, 12]. The role of immunosuppressives or immunomodulatory treatment remains uncertain [2]. Recently, Cheema et al. [11] suggested that infliximab therapy might be useful in reducing inflammation and leakage from the optic nerve according to their observation in 2 cases with treatment-resistant IRVAN syndrome.

Although the presence of anterior chamber cells and vitritis in most cases implies an underlying inflammatory etiology and thereby justifies corticosteroid treatment, systemic corticosteroids have not been shown to reduce the vasculitic process or halt the progression of retinal or iris neovascularization [4, 16]. An intravitreal dexamethasone implant can be deemed as a legitimate treatment alternative. Empeslidis et al. [13] administered a unilateral dexamethasone implant to combat persistent macular edema despite previous PRP, oral steroid treatment and pars plana vitrectomy in a patient with stage 3 IRVAN syndrome. Following the implant procedure, visual acuity had significantly improved, and the authors noted a marked reduction in macular thickness. The fellow eye received only PRP with a satisfactory stabilization. Sawhney et al. [15] employed PRP at the areas of retinal ischemia and aneurysmal dilations following 3 bevacizumab injections for an eye with stage 3 IRVAN syndrome. Over the ensuing 8 months, the patient was treated monthly with 7 additional bevacizumab injections, 1 intravitreal dexamethasone implant and 1 periocular triamcinolone injection. Pars plana vitrectomy was performed later on to excise the epiretinal membrane and relieve the vitreomacular traction. The macular lipid exudation had completely resolved with a residual lamellar hole.

Antivascular epidermal growth factor agents were mainly introduced to attack the neovascular process in addition to PRP with some success in patients with IRVAN syndrome [9, $10,16]$. However, their benefit as an adjunctive therapy for alleviating the exudative response has not been previously documented. In the present case, though PRP prevented the occurrence of ischemia-related complications, peripapillary exudates were not affected by PRP and antivascular epidermal growth factor treatment and even increased, but a single dexamethasone implant injection definitely contributed to the resolution of peripapillary exudation bilaterally.

In light of our case and recent publications, combination treatment seems to improve the anatomic and functional outcome in IRVAN syndrome, and intravitreal dexamethasone administration may play an adjunctive beneficial role.

\section{Disclosure Statement}

The authors declare that there are no conflicts of interest regarding the publication of this article. 
Saatci et al.: Single Bilateral Dexamethasone Implant in Addition to Panretinal Photocoagulation and Oral Azathioprine Treatment in IRVAN Syndrome

\section{References}

1 Chang TS, Aylward GW, Davis JL, Mieler WF, Oliver GL, Maberley AL, Gass JD: Idiopathic retinal vasculitis, aneurysms, and neuro-retinitis. Retinal Vasculitis Study. Ophthalmology 1995;102:1089-1097.

-2 Samuel MA, Equi RA, Chang TS, Mieler W, Jampol LM, Hay D, Yannuzzi LA: Idiopathic retinitis, vasculitis, aneurysms, and neuroretinitis (IRVAN): new observations and a proposed staging system. Ophthalmology 2007;114:1526-1529.

-3 Pichi F, Ciardella AP: Imaging in the diagnosis and management of idiopathic retinal vasculitis, aneurysms, and neuroretinitis (IRVAN). Int Ophthalmol Clin 2012;52:275-282.

-4 Yeshuran I, Recillas-Gispert C, Navarro-Lopez P, Arellanes-Garcia L, Cervantes-Coste G: Extensive dynamics in location, shape, and size of aneurysms in a patient with idiopathic retinal vasculitis, aneurysms, and neuroretinitis (IRVAN) syndrome. Idiopathic retinal vasculitis, aneurysms, and neuroretinitis. Am J Ophthalmol 2003;135:118-120.

5 Tomita M, Matsubara T, Yamada H, Takahashi K, Nishimura T, Sho K, Uyama M, Matsumura M: Long-term follow-up in a case of successfully treated idiopathic retinal vasculitis, aneurysms, and neuroretinitis (IRVAN). Br J Ophthalmol 2004;88:302-303.

6 Gedik S, Yilmaz G, Akça S, Akova YA: An atypical case of idiopathic retinal vasculitis, aneurysms, and neuroretinitis (IRVAN) syndrome. Eye 2005;19:469-471.

7 Venkatesh P, Verghese M, Davde M, Garg S: Primary vascular occlusion in IRVAN (idiopathic retinal vasculitis, aneurysms, neuroretinitis) syndrome. Ocul Immunol Inflamm 2006;14:195-196.

8 Ramli NM, Zahari M: IRVAN syndrome. Phillip J Ophthalmol 2007;32:46-48.

-9 Karagiannis D, Soumplis V, Georgalas I, Yandarakis A: Ranibizumab for idiopathic retinal vasculitis, aneurysms, and neuroretinitis: favorable results. Eur J Ophthalmol 2010;20:792-794.

10 Akesbi J, Brousseaud FX, Adam R, Rodallec T, Nordmann JP: Intravitreal bevacizumab (Avastin) in idiopathic retinitis, vasculitis, aneurysms and neuroretinitis. Acta Ophthalmol 2010;88:e40-e41.

11 Cheema RA, Al-Askar E, Cheema HR: Infliximab therapy for idiopathic retinal vasculitis, aneurysm, and neuroretinitis syndrome. J Ocul Pharmacol Ther 2011;27:407-410.

12 Rodrigues KFP, Lima VC, Arantes TEF, Matos KTK, Muccioli C: Retinite idiopática, vasculite, aneurismas e neurorretinite (IRVAN): relato de caso. Arq Bras Oftalmol 2012;75:140-142.

$\checkmark 13$ Empeslidis T, Banerjee S, Vardarinos A, Konstas AG: Dexamethasone intravitreal implant for idiopathic retinal vasculitis, aneurysms, and neuroretinitis. Eur J Ophthalmol 2013;23:757-760.

14 Rouvas A, Nikita E, Markomichelakis N, Theodossiadis P, Pharmakakis N: Idiopathic retinal vasculitis, arteriolar macroaneurysms and neuroretinitis: clinical course and treatment. J Ophthalmic Inflamm Infect 2013;3:21.

15 Sawhney GK, Payne JF, Ray R, Mehta S, Bergstrom CS, Yeh S: Combination anti-VEGF and corticosteroid therapy for idiopathic retinal vasculitis, aneurysms, and neuroretinitis syndrome. Ophthalmic Surg Lasers Imaging Retina 2013;44:599-602.

16 Basha M, Brown GC, Palombaro G, Shields CL, Shields JA: Management of IRVAN syndrome with observation. Ophthalmic Surg Lasers Imaging Retina 2014;45:e18-e22. 


\section{Case Reports in \\ Ophthalmology}

\begin{tabular}{l|l}
\hline \multicolumn{2}{l}{ Case Rep Ophthalmol 2015;6:56-62 } \\
\hline DOI: 10.1159/000375481 & $\begin{array}{l}\text { @ 2015 S. Karger AG, Basel } \\
\text { www.karger.com/cop }\end{array}$ \\
\hline
\end{tabular}

Saatci et al.: Single Bilateral Dexamethasone Implant in Addition to Panretinal Photocoagulation and Oral Azathioprine Treatment in IRVAN Syndrome

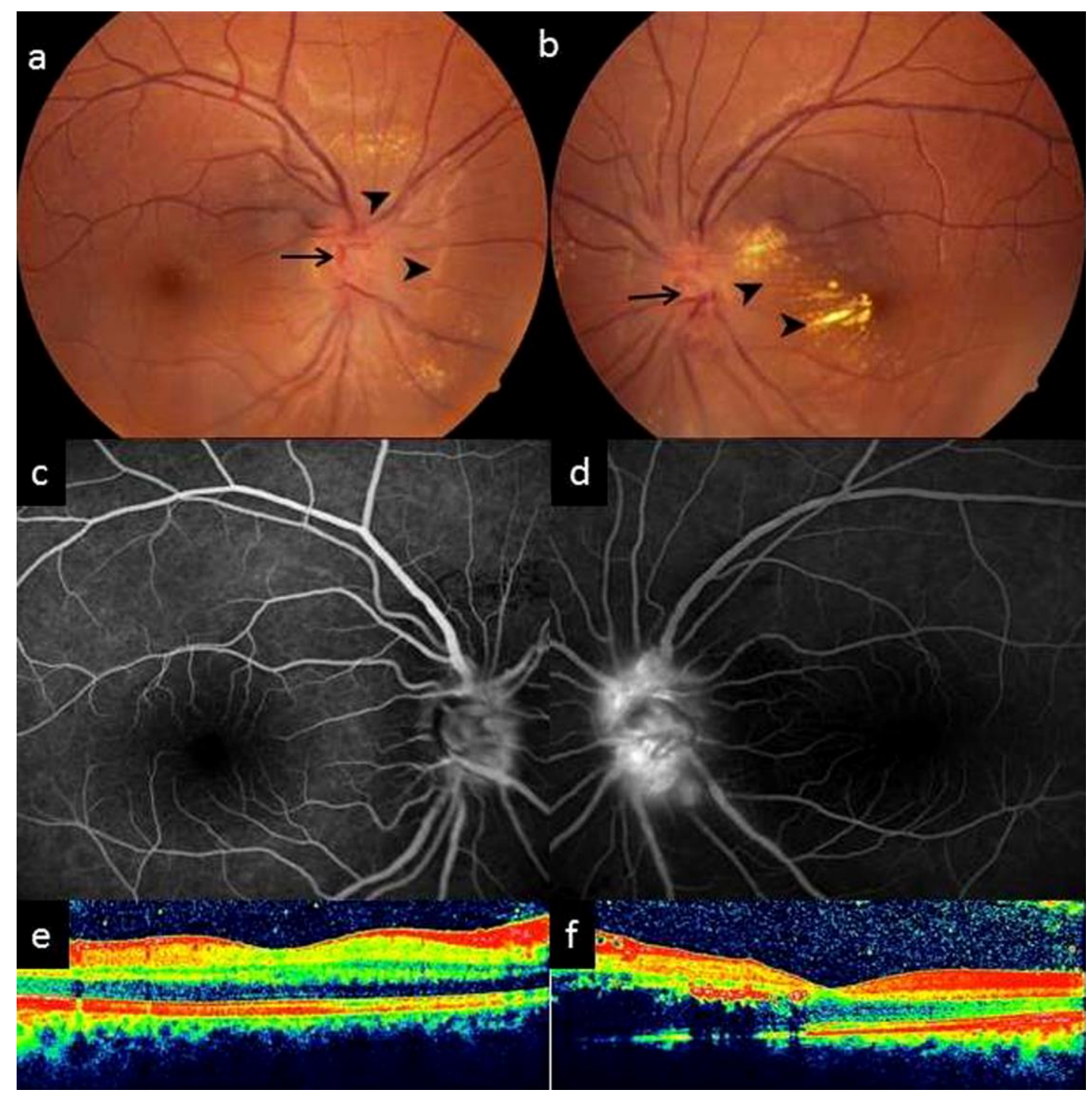

Fig. 1. a, b Color fundus picture of the OD (a) and OS (b) showing peripapillary exudates (arrowheads) and epipapillary vessel dilations (arrow) in December 2010. c, d Fluorescein angiogram depicting the disc staining in the OD (c) and OS (d). e, f OCT showing the near-normal right macula (e) and subretinal fluid with hyperreflective dots corresponding to hard exudates in the OS (f). 


\section{Case Reports in \\ Ophthalmology}

\begin{tabular}{l|l}
\hline \multicolumn{2}{l}{ Case Rep Ophthalmol 2015;6:56-62 } \\
\hline DOI: 10.1159/000375481 & $\begin{array}{l}\text { @ 2015 S. Karger AG, Basel } \\
\text { www.karger.com/cop }\end{array}$ \\
\hline
\end{tabular}

Saatci et al.: Single Bilateral Dexamethasone Implant in Addition to Panretinal Photocoagulation and Oral Azathioprine Treatment in IRVAN Syndrome

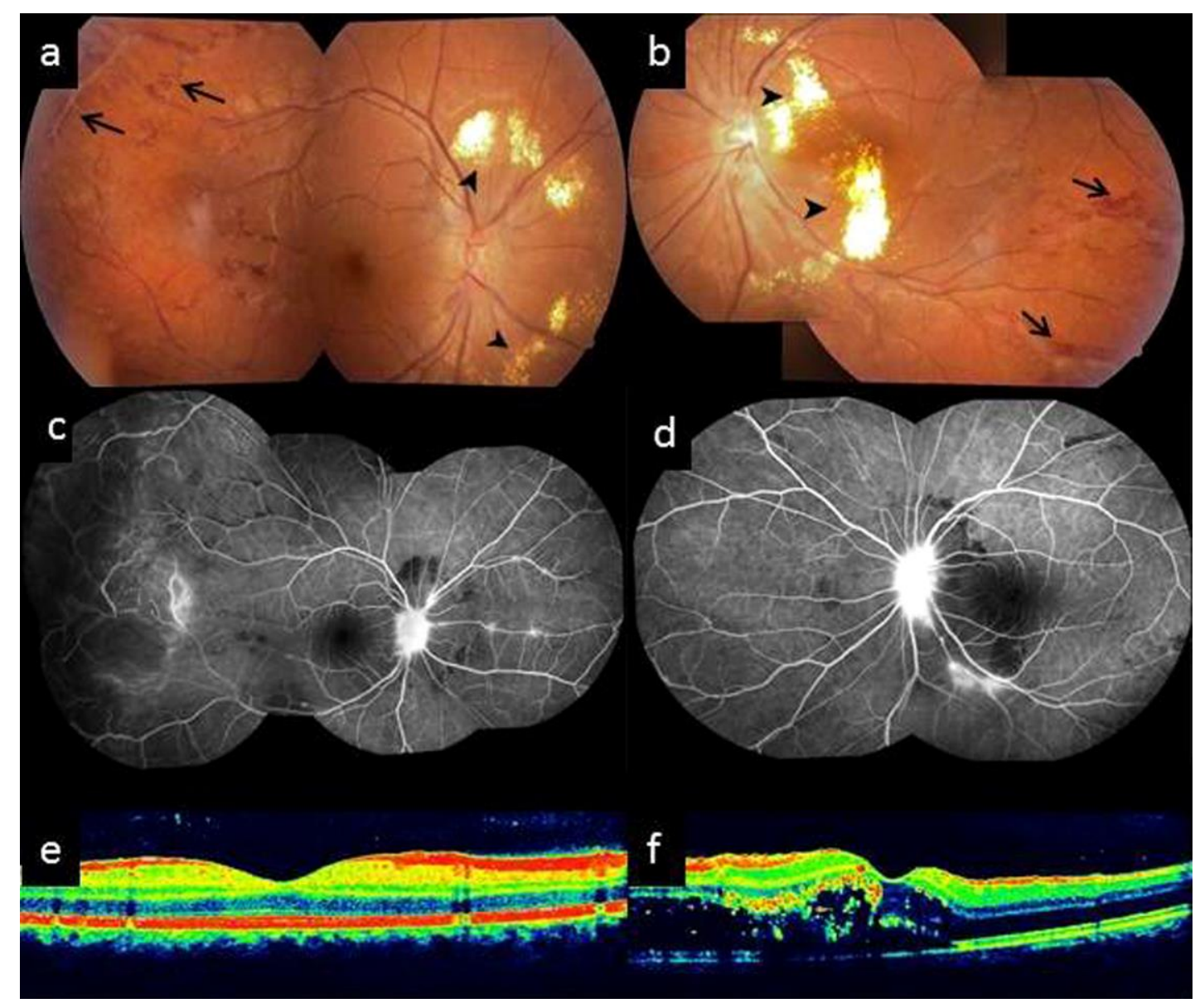

Fig. 2. a, b Color fundus picture of the OD (a) and OS (b) showing the persistent peripapillary exudates (arrowheads) with peripheral vasculitis (arrows) in April 2013, at the time when the diagnosis of IRVAN syndrome was established. c, d Fluorescein angiogram depicting $360^{\circ}$ severe peripheral capillary nonperfusion bilaterally in the OD (c) and OS (d). OCT revealing near-normal macula in the OD (e) and subretinal fluid with hyperreflective dots corresponding to hard exudates in the OS (f). 


\section{Case Reports in \\ Ophthalmology}

\begin{tabular}{l|l}
\hline \multicolumn{2}{l}{ Case Rep Ophthalmol 2015;6:56-62 } \\
\hline DOI: 10.1159/000375481 & $\begin{array}{l}\text { ○ 2015 S. Karger AG, Basel } \\
\text { www.karger.com/cop }\end{array}$ \\
\hline
\end{tabular}

Saatci et al.: Single Bilateral Dexamethasone Implant in Addition to Panretinal Photocoagulation and Oral Azathioprine Treatment in IRVAN Syndrome

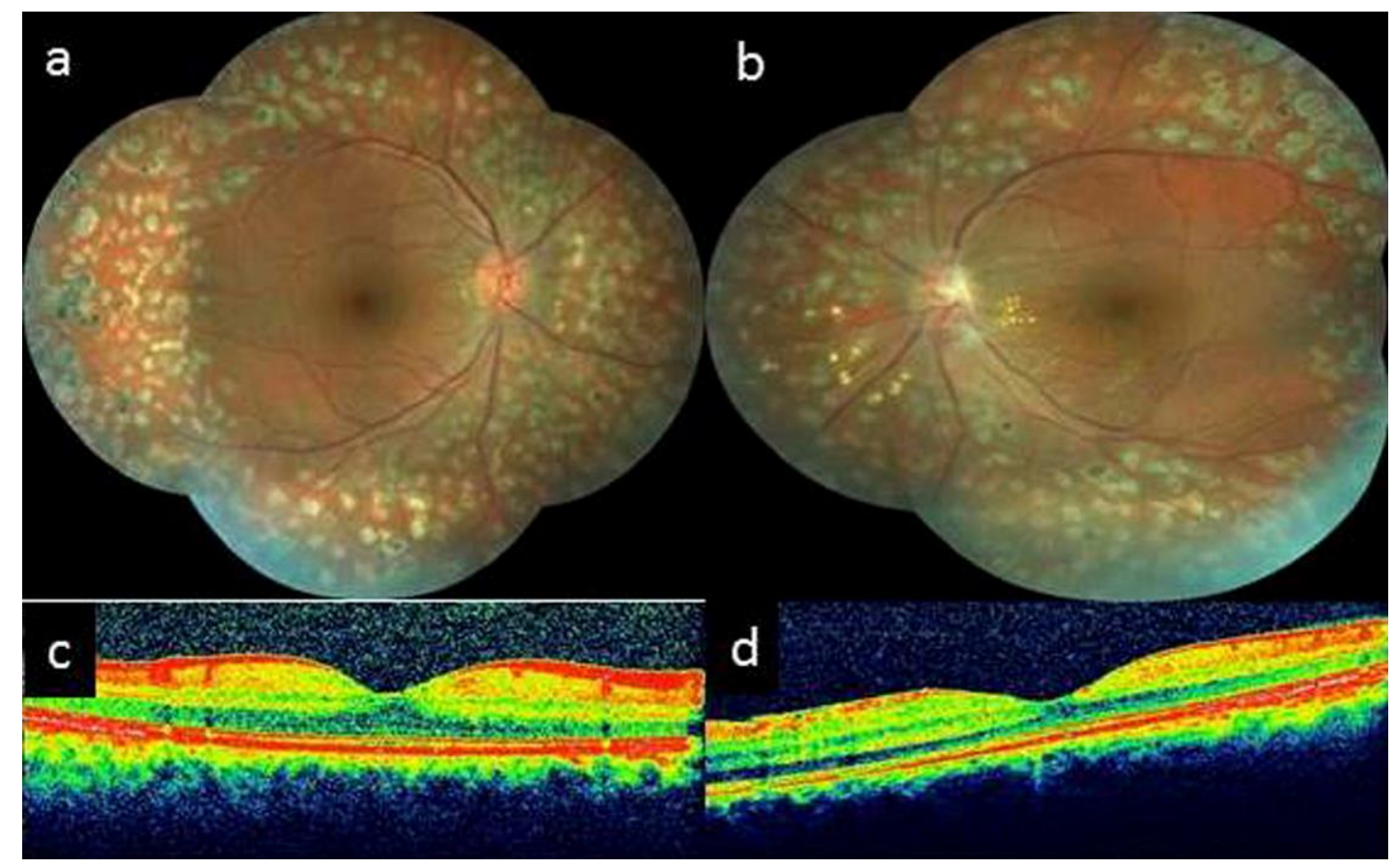

Fig. 3. a, b Color fundus picture of the OD (a) and OS (b) at the last visit in November 2014 depicting the resolution of peripapillary exudates with a stable fundus appearance. OCT exhibiting a flat posterior pole in the OD (c) and OS (d). 